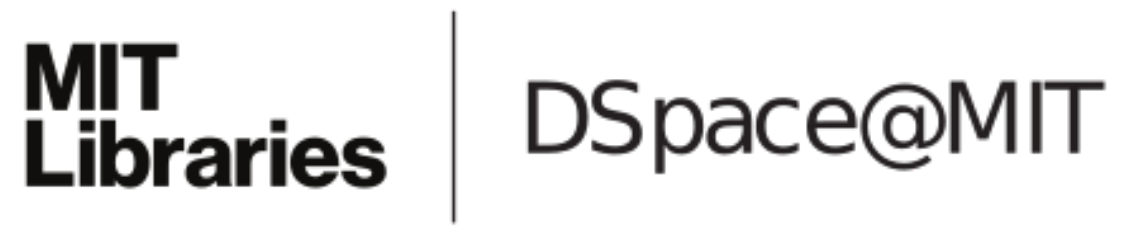

\author{
MIT Open Access Articles
}

IMPACT OF PICKUP IONS ON THE SHOCK FRONT NONSTATIONARITY AND ENERGY DISSIPATION OF THE HELIOSPHERIC TERMINATION SHOCK: TWODIMENSIONAL FULL PARTICLE SIMULATIONS AND COMPARISON WITH VOYAGER 2 OBSERVATIONS

The MIT Faculty has made this article openly available. Please share how this access benefits you. Your story matters.

Citation: Yang, Zhongwei, Ying D. Liu, John D. Richardson, Quanming Lu, Can Huang, and Rui Wang. “IMPACT OF PICKUP IONS ON THE SHOCK FRONT NONSTATIONARITY AND ENERGY DISSIPATION OF THE HELIOSPHERIC TERMINATION SHOCK: TWO-DIMENSIONAL FULL PARTICLE SIMULATIONS AND COMPARISON WITH VOYAGER 2 OBSERVATIONS." The Astrophysical Journal 809, no. 1 (August 5, 2015): 28. (C) 2015 The American Astronomical Society

As Published: http://dx.doi.org/10.1088/0004-637x/809/1/28

Publisher: IOP Publishing

Persistent URL: http://hdl.handle.net/1721.1/100050

Version: Final published version: final published article, as it appeared in a journal, conference proceedings, or other formally published context

Terms of Use: Article is made available in accordance with the publisher's policy and may be subject to US copyright law. Please refer to the publisher's site for terms of use. 


\title{
IMPACT OF PICKUP IONS ON THE SHOCK FRONT NONSTATIONARITY AND ENERGY DISSIPATION OF THE HELIOSPHERIC TERMINATION SHOCK: TWO-DIMENSIONAL FULL PARTICLE SIMULATIONS AND COMPARISON WITH VOYAGER 2 OBSERVATIONS
}

\author{
Zhongwei YAng ${ }^{1}$, Ying D. Liu ${ }^{1}$, John D. Richardson ${ }^{2}$, Quanming Lu ${ }^{3}$, CAn Huang $^{3}$, and Rui Wang ${ }^{1}$ \\ ${ }^{1}$ State Key Laboratory of Space Weather, National Space Science Center, Chinese Academy of Sciences, Beijing 100190, China; liuxying@spaceweather.ac.cn \\ ${ }^{2}$ Kavli Institute for Astrophysics and Space Research, Massachusetts Institute of Technology, Cambridge, MA 02139, USA \\ ${ }^{3}$ CAS Key Laboratory of Geospace Environment, Department of Geophysics and Planetary Science, University of Science and Technology of China, Hefei, China \\ Received 2015 April 22; accepted 2015 June 27; published 2015 August 5
}

\begin{abstract}
Voyager 2 (V2) observed multiple crossings of the heliospheric termination shock (TS) on 2007 August 31September 1 at a distance of 84 AU from the Sun. Here, for the first time, we present two-dimensional particle-incell (PIC) simulations of the TS self-consistently including pickup ions (PUIs), and compare the simulation results with $V 2$ observations. We find that (1) PUIs play a key role in the energy dissipation of the TS, and most of the incident ion kinetic energy is transferred to the thermal energy of PUIs. The PIC simulation indicates that, for the upstream parameters chosen for V2 conditions, the density of PUIs is about $25 \%$ and the PUIs gain the largest fraction (approximately 86.6\%) of downstream thermal pressure. (2) The simulated heliosheath ion distribution function is a superposition of a cold core formed by transmitted solar wind ions (SWIs), with the shoulders contributed by the hot reflected SWIs and directly transmitted PUIs, and the wings of the distribution dominated by the very hot reflected PUIs. The V2 Faraday cups observed the cool core of the distribution, and so they only saw the tip of the iceberg. (3) The nonstationarity of the shock front is mainly caused by ripples along the shock front which form even if the percentage of PUIs is high. These simulation results agree reasonably well with the V2 experimental data. The relevance of the shock front ripples to the multiple TS crossings observed by $V 2$ is also discussed in this paper.
\end{abstract}

Key words: interplanetary medium - plasmas - shock waves - Sun: heliosphere

\section{INTRODUCTION}

The solar wind blows outward from the Sun and forms a bubble of solar material in the interstellar medium. Because the interstellar plasma confines the solar wind, it has to become subsonic before directly interacting with the interstellar plasma, and this transition occurs at the heliospheric termination shock (TS; Decker et al. 2005; Burlaga et al. 2008; Jokipii 2008; Richardson et al. 2008). Interstellar neutrals enter the heliosphere and are ionized by charge exchange with the solar wind ions (SWIs; Vasyliunas \& Siscoe 1976; Möbius et al. 1985; Lee \& Ip 1987; Galeev \& Sagdeev 1988). They are then picked up by the solar wind and are referred to as pickup ions (PUIs). After these PUIs are convected to the TS, they are energized and a fraction of them become anomalous cosmic rays (ACRs) due to diffusive shock acceleration (Axford et al. 1977; Bell 1978; Blandford \& Ostriker 1978; Giacalone 2005; Fisk et al. 2006; Zank et al. 2006; Guo \& Giacalone 2010). The TS is strongly influenced by the presence of PUIs (Liewer \& Goldsten 1993; Gloeckler \& Geiss 1998; Matsukiyo et al. 2007), which makes the TS very different from planetary and interplanetary shocks inside the heliosphere (Lembège et al. 2004; Burgess et al. 2005; Goncharov et al. 2014). Of particular interest are the microstructure and the energy dissipation of the TS.

First, the number density of PUIs at the TS is relatively high ( 25\%; Richardson et al. 2008; Wu et al. 2009), and thus may greatly modify the microstructure of the shock front. The TS was expected to be a boundary that is stable on a timescale of several days. The Voyager 2 (V2) plasma experiment observed a decrease in the solar wind speed commencing on about 2007 June 9, which culminated in several observations of TS crossings (named TS-2, TS-3, and TS-4) between 2007 August
30 and September 1 (Burlaga et al. 2008; Decker et al. 2008; Richardson et al. 2008; Stone et al. 2008). At least two TS crossings (TS-1 and TS-5) occurred when there were gaps in the telemetry. Observations of the magnetic field structure and dynamics of the TS were reported (Burlaga et al. 2008). The TS location depends on the solar activity, and it moves inward and outward due to changes in the solar wind pressure on a long timescale (several years; Whang \& Burlaga 2000). However, the underlying mechanisms causing the V2 spacecraft to make so many crossings in such a short time (at least 5 in 2.7 days, with separations as small as $3 \mathrm{hr}$ ) still remain unclear. It is believed that the multiple crossings imply motion of the TS, which would be caused by shock front nonstationarity (Burlaga et al. 2008).

There are many types of nonstationarity for a shock front, e.g., self-reformation (Lembège \& Dawson 1987; Hada et al. 2003; Chapman et al. 2005; Matsukiyo \& Scholer 2014), self-excited ripples (Winske \& Quest 1988; Savoini \& Lembège 1994; Lembège et al. 2004; Burgess \& Scholer 2007), and pre-existing waves or turbulence (Giacalone 2005; Guo \& Giacalone 2010), some of which likely cause the unexpected motions of the TS. These nonstationarities are predicted and observed by numerical simulations and satellite observations, respectively. The term "self-reformation" describes a process wherein the particles reflected by the shock ramp accumulate ahead of the shock and form a shock foot, which then grows and becomes the new ramp. The new ramp starts to reflect incident particles, and the process repeats. Self-reformation of the shock front was predicted by both hybrid simulations (Tiu et al. 2011; Hellinger \& Trávnícek 2002; Lembège et al. 2009; Yuan et al. 2009; Su et al. 2012) and particle-in-cell (PIC) simulations (Lembège \& Dawson 1987; Hada et al. 2003; 
Nishimura et al. 2003; Scholer et al. 2003; Lee et al. 2005a; Yang et al. 2009, 2013) for large Mach number and low $\beta_{i}$ shocks, where $\beta_{i}$ is the ratio of the thermal pressure of ions to the magnetic pressure. Even in the presence of PUIs, selfreformation occurs under some conditions (Chapman et al. 2005; Lee et al. 2005b; Oka et al. 2011; Yang et al. 2012a; Matsukiyo \& Scholer 2014). For the heliospheric TS, the values of $\beta_{i}$ and the Mach number are relatively low and high, respectively. The TS is generally believed to be in the supercritical regime and probably undergoes self-reformation (Burlaga et al. 2008). Self-excited ripples are usually found in two-dimensional (2D) hybrid simulations (Thomas 1989; Burgess \& Scholer 2007) and PIC simulations (Savoini \& Lembège 1994; Lembège et al. 2009; Yang et al. 2012b). Shock front ripples are robust in three-dimensional (3D) hybrid simulations (Thomas 1989; Hellinger et al. 1996). The filament instability of the shock front found in high-dimensional simulations can also contribute to the shock front nonstationarity (Spitkovsky 2005; Guo \& Giacalone 2013; Caprioli \& Spitkovsky 2014). Not all of the simulations above include PUIs. By using the 2D Los Alamos hybrid simulation code with PUIs, Liu et al. (2010) studied the Alfvén-cyclotron and mirror modes excited in the near-TS heliosheath. The impact of the PUIs on the shock front ripples and self-reformation is not mentioned. It is expected that the density of PUIs at the TS is of the order of $20 \%-30 \%$ of the solar wind density (Richardson et al. 2008; Wu et al. 2009; Matsukiyo \& Scholer 2014). The impact of such a high percentage of PUIs on the shock front ripples has not been studied yet. The relevance of the shock front ripples to the multiple TS crossings still remains unclear. A precise description of this influence would require at least a 2D full particle model.

Second, the high percentage of PUIs may greatly change the dissipation mechanism of the shock front. The $V 2$ has a working plasma instrument with sufficient coverage to identify three crossings of the TS. The TS- 3 crossing revealed an almost classical perpendicular shock structure (Burlaga et al. 2008; Richardson et al. 2008). The TS heats the incident SWIs very little, and the average downstream ion temperature is much smaller than predicted by the MHD Rankine-Hugoniot conditions. Richardson et al. (2008) concluded that most of the solar wind energy is transferred to PUIs or other energetic particles that reside in the energy range not covered by the $V 2$ plasma instrument. Zank et al. (1996) predicted that the TS dissipation mechanism would favor PUIs and leave the SWIs relatively cool. They concluded that PUIs may therefore provide the primary dissipation mechanism for a perpendicular TS with SWIs playing a secondary role. Previous onedimensional (1D) hybrid simulations with multiple ion species (Wu et al. 2009) suggest that the density of PUIs is about $25 \%$ and the PUIs gain the largest fraction (approximately 90\%) of the downstream thermal pressure. They defined a downstream pressure ratio $\chi=P_{\mathrm{t}}^{\mathrm{PUI}} / P_{\mathrm{t}}$, where $P_{\mathrm{t}}^{\mathrm{PUI}}$ and $P_{\mathrm{t}}$ represent the thermal pressure of PUIs and the total ions, respectively. However, it is not clear how the upstream plasma dynamic energy transfers to the PUIs inside the shock front or how the downstream pressure ratio $\chi$ varies in different locations along the shock surface (i.e., a 2D effect). In order to understand the energy dissipation process and the energy partition between different species of ions, here we develop a $2 \mathrm{D}$ full particle model of the TS.
It is also worth studying the velocity distribution function downstream of the TS using PIC simulations that include PUIs. The Voyager spacecraft are making in situ measurements along two different trajectories in the heliosheath, but unfortunately they were not designed to measure PUIs directly. IBEX was launched on 2008 October 19 (McComas et al. 2009) and is measuring the energetic neutral atom (ENA) flux from the boundary region of the heliosphere. The interpretation of ENA fluxes measured at $1 \mathrm{AU}$ by $I B E X$ requires knowledge of the ion velocity distribution function in the inner heliosheath. ENAs are created through the charge exchange of interstellar neutrals with hot heliosheath protons or ions. The flux of ENAs therefore depends sensitively on the number of hot protons downstream of the TS. Heerikhuisen et al. (2008) find that the ENA flux for a $\kappa$ distribution is higher than that for a Maxwellian proton distribution with the same temperature. This is not surprising because the $\kappa$ distribution contains many more particles in the wings of the distribution function than the corresponding Maxwellian distribution. Why the heliosheath proton distribution function should be a $\kappa$ distribution is, however, unclear. The answer may well reside in the processing of the upstream PUI distribution by the TS and the subsequent relaxation of the processed distribution in the heliosheath.

In this paper, we use a 2D PIC code to investigate the impact of PUIs on the shock front microstructure, the energy dissipation, and the downstream particle velocity distribution function of the TS. This paper is organized as follows. The simulation model is described in Section 2. We describe the simulation results in Section 3, focusing on the impact of PUIs on the shock front nonstationarity and the particle energy partition. In Section 4, we compare the simulation results with V2 observations. Finally, conclusions are drawn in Section 5.

\section{SIMULATION MODEL}

We use a 2D electromagnetic PIC code to simulate the evolving structures of supercritical, collisionless, perpendicular shocks with PUIs. Simulations of nonstationary shocks have been performed by 1D PIC codes including PUIs (Chapman et al. 2005; Matsukiyo \& Scholer 2011; Yang et al. 2012a). In this paper, we expand our simulation code to two dimensions in order to investigate the ripples of the shock front. Here, the control equations of the PIC code are only the Maxwell and Newton-Lorentz equations. Due to the numerical error built up during PIC simulations, the Gauss law cannot be assumed to be satisfied all of the time. Instead of solving the Poisson equation, our code solves only two curls, i.e., the Ampere and Faraday equations. A rigorous charge conservation method for the current deposits is described in Villasenor \& Buneman (1992). This charge conservation method requires that an additional equation, $\partial \rho / \partial t=-\nabla \cdot \boldsymbol{J}$, be solved at each time step to provide the current density to the field update. This method has commonly been used in previous PIC simulations (Buneman et al. 1995; Nishikawa 1997; Cai et al. 2003; Spitkovsky 2008; Rekaa et al. 2014). The particle data are updated by the leapfrog method (Birdsall \& Langdon 1991).

For the 2D simulations, we consider a Cartesian grid $(x, y)$. The plasma box sizes along the shock normal and shock front are $L_{x}=4096 \Delta_{x}$ and $L_{y}=512 \Delta_{y}$, respectively, where the numerical grid spacing is $\Delta_{x}=\Delta_{y}=0.025 c / \omega_{p i}=0.25 c / \omega_{p e}$. The spatial resolution is high enough to resolve the microstructures of the shock front even on the electron inertial scale 
(Mazelle et al. 2010). The physical vectors, such as the velocities of particles and electromagnetic fields, have components in three directions and spatially depend on $x$ and $y$. A shock is produced using the so-called piston method (Burgess \& Scholer 2007; Hao et al. 2014), in which the plasma is injected continuously from one end of the simulation box $(x=0$, in our case) and reflected elastically at the other end $\left(x=L_{x}\right)$. The upstream plasma has a uniform density of 16 particles per species per cell. The fractions of the particles of different species can be changed for different purposes (e.g., for a 25\% PUI case, the fractions of electrons, SWIs, and PUIs are 1, 0.75, and 0.25, respectively). The right boundary is assumed to be a perfectly conducting barrier. The pileup of the density and magnetic field creates a shock propagating in the $-x$ direction. In the 2D simulation, the boundary conditions of the electromagnetic fields in the $y$ direction are periodic, and the particles that move out of one end of the simulation domain in the $y$ direction will re-enter the domain from the other end. The initial distribution functions for the SWIs and electrons are both Maxwellian. PUIs are injected in a thin sphere in velocity space centered at $V_{\text {inj }}$ with a radius $V_{\text {shell }}$ as in earlier 1D works (Chapman et al. 2005; Yang et al. 2012a; Matsukiyo \& Scholer 2014). The upstream Alfvén speed is $V_{\mathrm{A}}=1$. To reduce the computational time, we use an unrealistic mass ratio for ions and electrons $m_{i} / m_{e}=100$ and a light speed of $c=20 V_{\mathrm{A}}$ as in previous work (Chapman et al. 2005; Oka et al. 2011). The basic parameters and configuration are as follow. The ambient magnetic field $B_{o}$ is in the $Y$ direction as in previous work (Winske \& Quest 1988; Liu et al. 2010). The SWI $\beta_{i}$ is 0.04 as observed by Voyager 2 (Burlaga et al. 2008; Richardson et al. 2008). The injected plasma is quasi-neutral, i.e., $n_{i}=n_{e}$ and $n_{i}=n_{\mathrm{SWI}}+n_{\mathrm{PUI}}$, where $n_{e}, n_{i}, n_{\mathrm{SWI}}$, and $n_{\mathrm{PUI}}$ are densities of the electrons, total ions, SWIs, and PUIs, respectively.

\section{SIMULATION RESULTS ON THE IMPACT OF PUIs}

In this section, the impact of PUIs on the shock front nonstationarity will be analyzed in detail for three cases with different percentages of PUIs (PUI\%): 0\% (Run A), 10\% (Run $\mathrm{B}$ ), and $25 \%$ (Run C). Then, we concentrate on how the upstream ion dynamic energy transfers to the thermal energy of PUIs and SWIs and the magnetic energy within the shock transition layer. The energy partition of SWIs and PUIs downstream of the TS is computed as in previous 1D hybrid simulations (Wu et al. 2009).

First, we investigate the impact of the relative percentage of PUIs on the shock front. Figure 1 is an overview of Runs A $(\mathrm{PUI} \%=0), \mathrm{B}(\mathrm{PUI} \%=10)$, and C $(\mathrm{PUI} \%=25)$. In each panel, the surface indicates the magnetic field $B$ in the $2 \mathrm{D}$ simulation domain at a time $t=4 \Omega_{c i}^{-1}$. Figure 1 (a) shows that in the absence of PUIs, the shock front is characterized by selfexcited ripples (marked by a red box) as in previous 2D PIC simulations without PUIs (Savoini \& Lembège 1994; Lembège et al. 2009). Typical structures of a supercritical perpendicular shock such as the foot ("F"), ramp ("R"), and overshoot ("O") are evident (see the magnetic field profile at $Y=0$ marked by the black solid curve). The upstream Alfvénic Mach number $M_{\mathrm{A}}$ is about 4.5. A similar plot for Run B is shown in Figure 1(b). In this case, a weak but broad PUI foot with an average amplitude of $\sim 1.15 B_{o}$ (marked by "PUI F") emerges ahead of the SWI foot (marked by "SWI F"). The PUI foot is stationary. Figure 1(c) shows the corresponding plot for Run C. With PUI\% $=25$, the amplitude of the PUI foot becomes
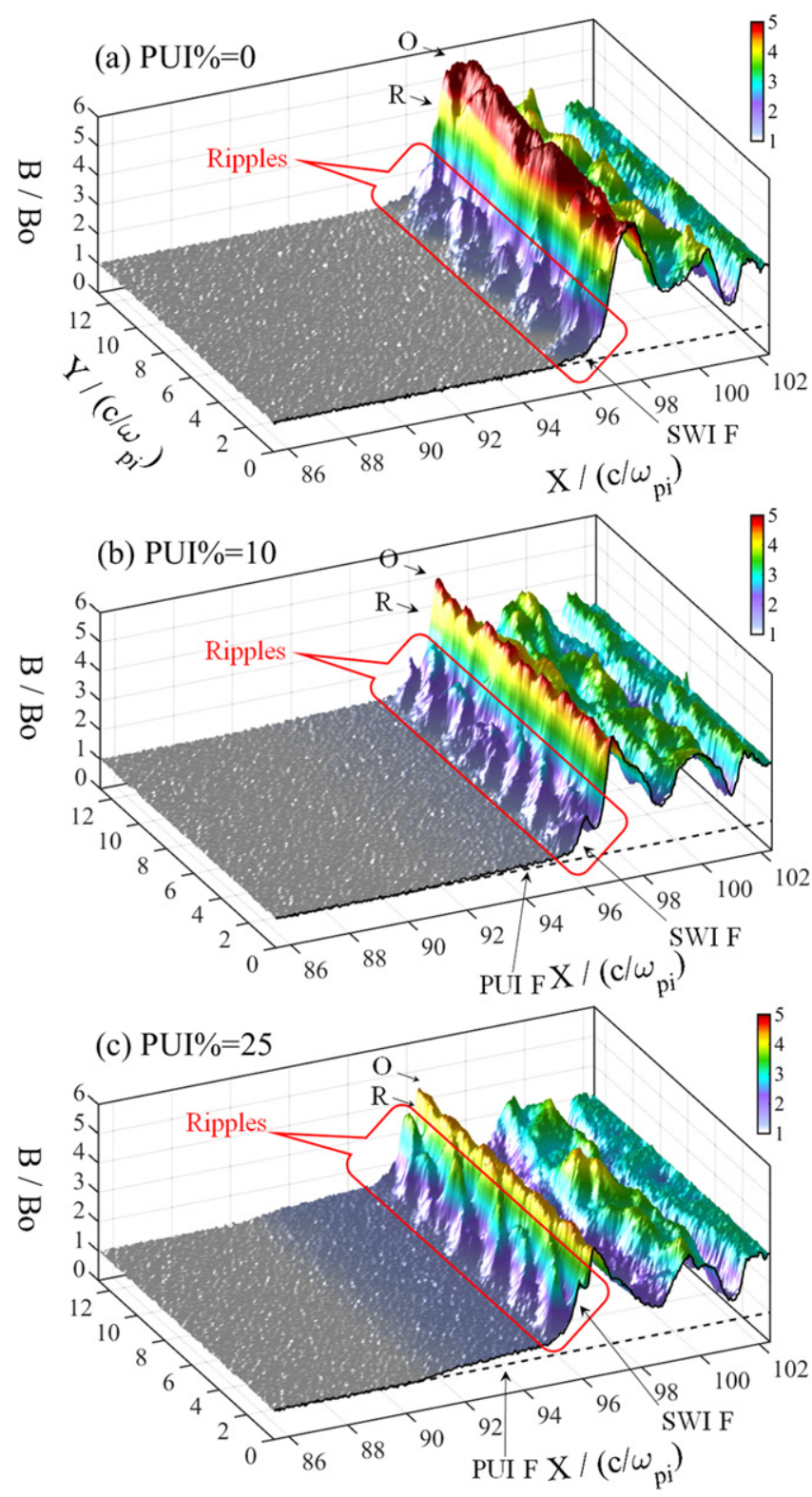

Figure 1. Overview of the magnetic field profiles at a time $t=4 \Omega_{c i}^{-1}$ for (a) PUI $\%=0$, (b) PUI $\%=10$, and (c) PUI $\%=25$. The color shading indicates the strength of the magnetic field. The shock foot, ramp, and overshoot are marked by "F," "R," and "O," respectively. The foot regions due to the reflected PUIs and SWIs are labeled as "PUI F" and "SWI F," respectively. The dashed black reference line shows the upstream value $B_{o}$. The $B$ profiles at $Y=0$ are shown by black solid curves. The shock front ripples are marked by a red box.

higher but the amplitude of the overshoot becomes lower than that in Runs A and B.

As can be seen from Figure 1, the shock front is nonstationary even in the high PUI\% case due to the selfexcited ripples. The impact of the relative percentage of PUIs on the rippling shock front can be illustrated from two aspects: wave features and particle behavior. Figure 2 plots the corresponding power spectrum of the fluctuating magnetic field $B$. The color shading indicates the power $\left|B\left(k_{y}\right)\right|^{2}$ that is obtained by Fourier transforming the values of $B$ along the $Y$ direction (i.e., along the shock front) at a selected position $X$. In order to show the location of the shock front, the $Y$-averaged magnetic field $B / 3$ has been overlaid on the spectrum for 

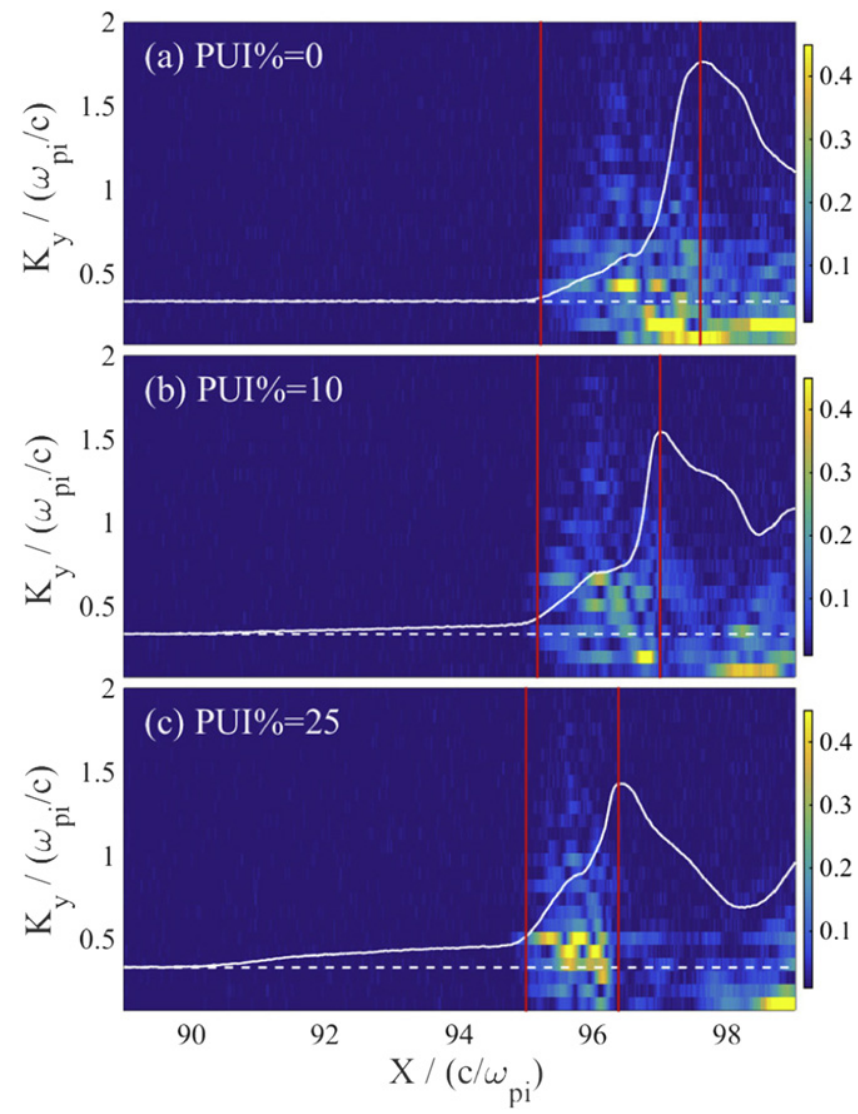

Figure 2. Power spectrum of the fluctuating magnetic field $B$ across the shock with $0 \%, 10 \%$, and $25 \%$ PUIs (from top to bottom) at $t=4 \Omega_{c i}^{-1}$. The color shading indicates the power $\left(\left|B\left(K_{y}\right)\right|^{2}\right)$ that is obtained by Fourier transforming the values of $B$ along the $Y$ direction at a selected position $X$. The vertical red lines denote the interval of the rippling region. The profile $\bar{B} / 3$ (averaged along the $Y$ direction) is superimposed.

reference (white curve). The horizonal dashed line indicates the upstream value of $B / 3$. The magnetic field fluctuations are enhanced greatly from the SWI foot to the ramp. With the increase in PUI\%, the ripple excitation region in the $x$ direction (between the two vertical red lines) becomes narrower.

To understand why the scale of ripples along $Y$ decreases with the relative percentage of PUIs, we perform particle diagnosis for the cases in Figure 2. Corresponding phase space diagrams $\left(X-V_{i x}\right.$, black dots $)$ of SWIs are plotted in the downstream rest frame (i.e., the simulation frame) in Figure 3. The $Y$-averaged magnetic field $B$ is also shown for reference (blue curve). A fraction of the incident SWIs coming from the left-hand side is reflected at the shock front, and the reflected ions become a hot SWI population when convected back to the downstream. The other incident SWIs are directly transmitted to the downstream region and form the cool core of the downstream ion velocity distribution. The shock front ripple is associated with the reflected SWIs. Briefly, the magnetic field of the PUI foot ahead of the shock ramp increases with the percentage of PUIs. In a high PUI\% case, the gyroradius of the reflected SWIs becomes smaller due to the enhanced local $B$ caused by the reflected PUIs at the foot. This smaller gyroradius leads to a narrower ripple excitation region at the shock front.

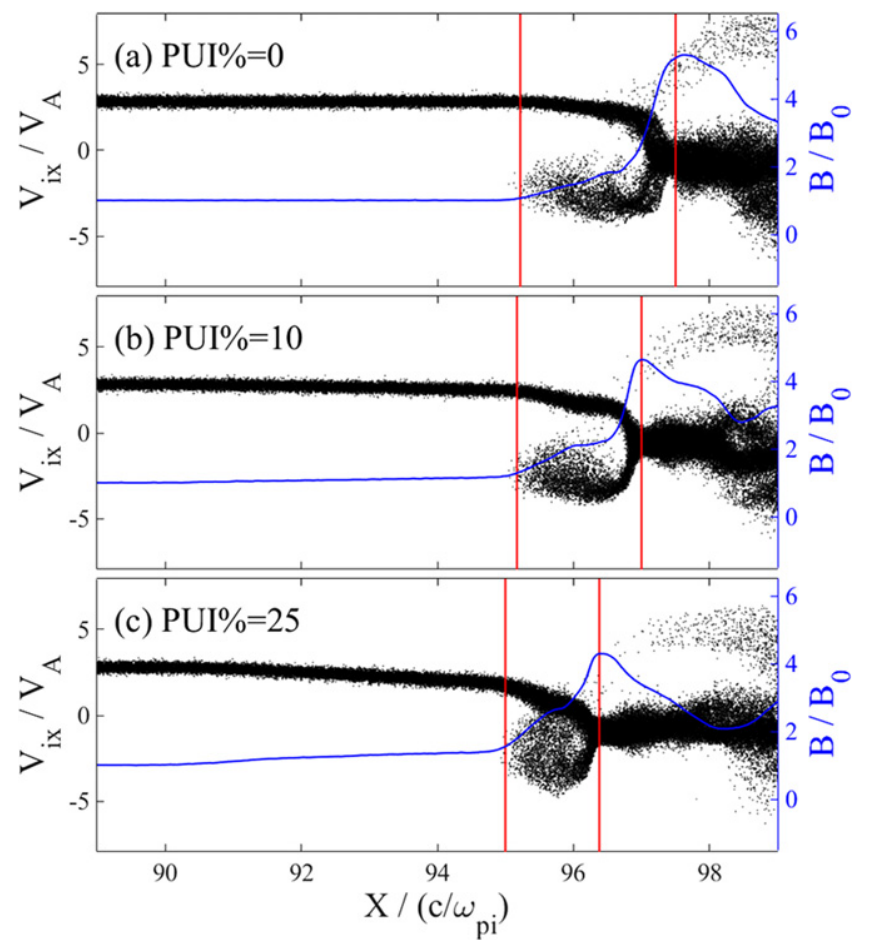

Figure 3. Phase space plots $\left(V_{i x}\right.$ vs. $\left.X\right)$ of SWIs at $t=4 \Omega_{p i}^{-1}$. From top to bottom, the relative densities of the PUIs are $0 \%, 10 \%$, and $25 \%$, respectively. In each panel, the $Y$-averaged $B$ field (blue curve) is shown for reference. The ripple region is between the two vertical red lines.

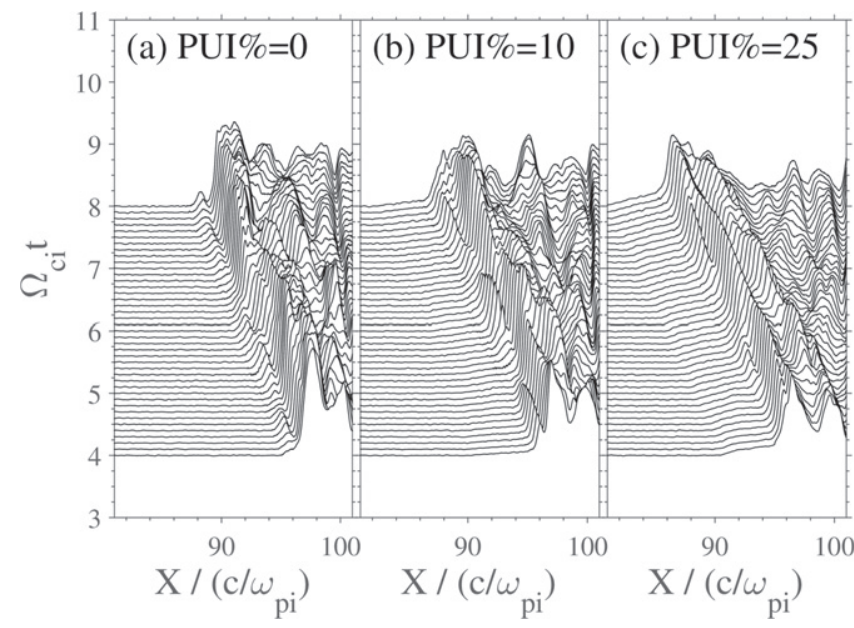

Figure 4. Stack plots of the $B$ profiles (at $Y=0$ ) at different times. From left to right, the relative density of PUIs is $0 \%, 10 \%$, and $25 \%$, respectively.

Figure 4 shows stack plots of the $B$ profiles at a fixed $Y(=0)$ versus time with different PUI\%. The period of the shock front nonstationarity is about $1-2 \Omega_{c i}^{-1}$, which is close to the selfreformation time observed in 1D PIC simulations (Hada et al. 2003; Chapman et al. 2005; Yang et al. 2009). The timescale of the first TS crossing (time between two ramps in TS-2) observed by $V 2$ is about 29 minutes (Richardson et al. 2008), which is equivalent to $1.7 \Omega_{c i}^{-1}$. Our PIC simulation gives a similar period, and so TS-2 is likely caused by the selfexcited rippling at the shock front. The time intervals of two other crossings (TS-3 and TS-4) are about 3.7 and $2.8 \mathrm{hr}$ $\left(\sim 10 \Omega_{c i}^{-1}\right)$, respectively. These crossings are likely due to the 
interaction of the TS with pre-existing waves or turbulence with a larger temporal scale (Guo \& Giacalone 2012).

Second, we study the energy dissipation process within the shock transition layer and the resulting energy partition in the downstream region of the TS. Wu et al. (2009) studied the energy partition of PUIs and SWIs at the TS using 1D hybrid simulations. For comparison with $V 2$ observations, they defined the energy partition of PUIs in the downstream as a pressure ratio $\chi=P_{\mathrm{t}}^{\mathrm{PUI}} / P_{\mathrm{t}}$, where $P_{\mathrm{t}}$ and $P_{\mathrm{t}}^{\mathrm{PUI}}$ are the downstream thermal pressure of the total ions and PUIs, respectively. The pressure ratio $\chi$ increases as the PUI relative density increases. In the high percentage $(25 \%)$ PUI case, the ratio $\chi$ is about $90 \%$, which is the energy fraction gain for PUIs inferred from the Voyager 2 observations by Richardson et al. (2008). However, 1D hybrid simulations have the fundamental limitation that downstream heating occurs only in the two directions perpendicular to the magnetic field (Wu et al. 2009). Two-dimensional hybrid simulations of quasi-perpendicular shocks without PUIs have demonstrated downstream ion temperature anisotropies and associated cyclotron and mirrorlike fluctuations (Winske \& Quest 1988; Lu \& Wang 2006; Hao et al. 2014), consistent with observations (Liu et al. 2006, 2007; Richardson 2007). Here, we examine the energy partition of PUIs in the downstream of the TS by using a 2D PIC code which allows the heating to take place in all three directions.

For reference, we present the energy dissipation at a shock without PUIs. Figure 5 shows the phase space plots $\left(X-V_{i x}, V_{i y}\right.$ and $V_{i z}$ ) of SWIs in the shock rest frame for Run A. Note that the simulations are performed in the downstream rest frame so that the downstream flow speed $\left|V_{\mathrm{DS}}\right|=\left|V_{\mathrm{s}}\right|$, where $V_{\mathrm{s}}$ is the shock propagation speed. The top three panels in Figure 5 show that the SWIs are mainly heated along the directions perpendicular to the magnetic field. The dynamic pressure of the SWIs along the shock normal can be calculated from $P_{d}=n m V_{x}^{2}$, where $n, m$, and $V_{x}$ are the density, mass, and bulk velocity of ions in the $X$ direction. The thermal pressure of SWIs along the shock normal can be calculated from $P_{\mathrm{t}}=n k T$, where $k$ and $T$ are the Boltzmann constant and the temperature of the particles. The magnetic pressure $P_{\mathrm{b}}$ is computed from $B^{2} /\left(2 \mu_{0}\right)$. All of the pressures are normalized by $n_{0} m_{0} V_{\mathrm{A}}^{2}$ of the upstream region (Figure $5(\mathrm{~d})$ ), where $m_{0}$ and $n_{0}$ are the mass and upstream density of the ions, respectively. A reduction of the dynamic pressure of SWIs is evident at the shock transition. Both $P_{\mathrm{t}}$ and $P_{\mathrm{b}}$ increase at the the shock transition, and their sum is negatively correlated with the dynamic pressure $P_{\mathrm{d}}$. Obviously, the upstream dynamic energy is transferred to the SWI thermal energy and magnetic energy in the shock transition.

The impact of PUIs on the dissipation process and the energy partition is shown in Figure 6 with a moderate PUI\% (10). In the PUI foot region (from $X=84 \mathrm{c} / \omega_{p i}$ to about $\left.X=89 c / \omega_{p i}\right)$, the dynamic pressures of both PUIs and SWIs start to decrease. Simultaneously, the thermal pressure of PUIs increases due to the reflected PUIs (Figure 6(a)). There are no reflected SWIs in the PUI foot (see Figure 6(a)), and thus the thermal pressure of SWIs is almost unchanged in this region. The magnetic pressure $P_{\mathrm{b}}$ slightly increases at the PUI foot. Therefore, most of the decreased dynamic energy of the incident ions (PUIs plus SWIs) is transferred to the PUIs as thermal energy in the PUI foot. In the SWI foot region (from $X=89 c / \omega_{p i}$ to about $\left.X=90.25 c / \omega_{p i}\right)$, the dynamic pressure
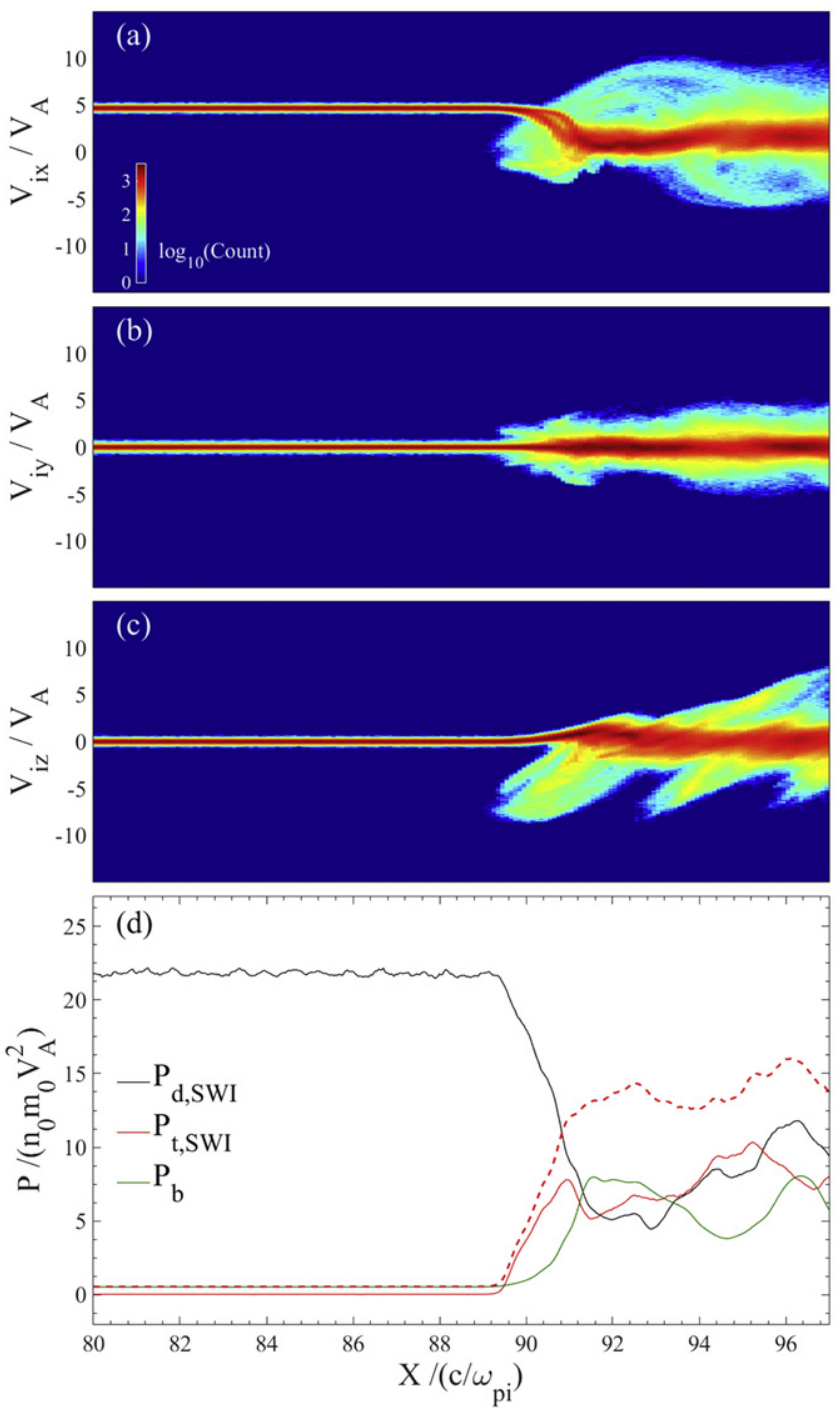

Figure 5. (a) Phase space plots $\left(X-V_{i x}\right)$ of the SWIs at $t=7 \Omega_{c i}^{-1}$ in Run A $(\mathrm{PUI} \%=0)$. The color shading shows logarithmic distributions of the particle number. (b)-(c) Similar plots, but for $X-V_{i y}$ and $X-V_{i z}$, respectively. (d) The dynamic pressure of SWIs (black), the thermal pressure of SWIs (red), and the magnetic pressure (green) across the shock front. The sum of the SWI thermal pressure and the magnetic pressure is also shown for reference (red dashed).

of SWIs decreases drastically due to the reflected SWIs, while the dynamic pressure of PUIs is almost unchanged. The thermal pressure of PUIs and the magnetic field pressure increase gradually. Thus, most of the energy of SWIs dissipates to the thermal energy of SWIs in the SWI foot. In the narrow ramp region (from $X=90.25 \mathrm{c} / \omega_{p i}$ to the about $\left.X=90.75 \mathrm{c} / \omega_{p i}\right)$, all of the pressures decrease except for the magnetic pressure, which shows a steep increase. Thus, the dynamic energy and the thermal energy of the ions are transferred to magnetic energy in this region. Figure 6(c) shows the thermal pressure ratio $\chi$ in the downstream region. Curves in different colors indicate the $\chi$ profiles obtained in different $Y$ locations. The ratio $\chi$ can vary versus $Y$ due to the downstream turbulence as a remnant of the shock front ripples (a 2D effect). This result cannot be obtained from 1D simulations because $\partial / \partial Y=0$. The average value of the ratio 


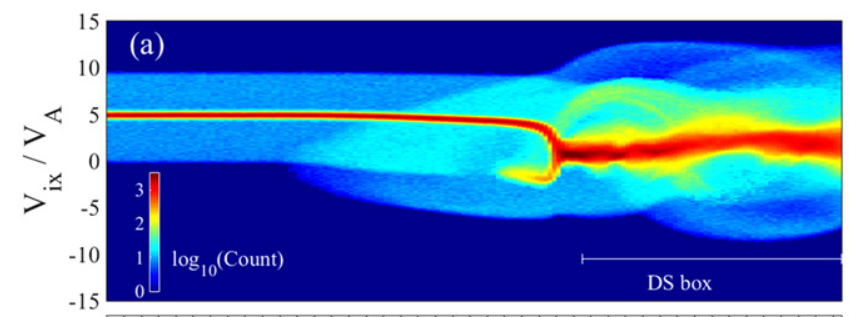

(b)
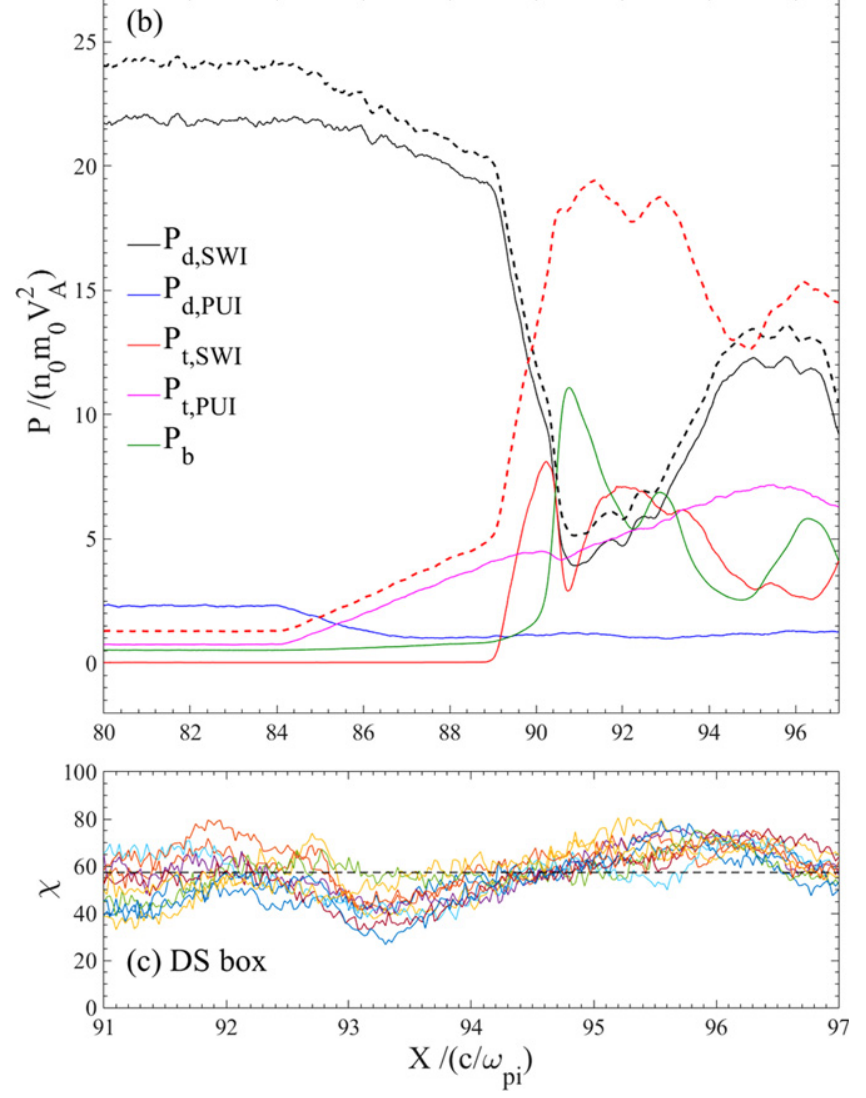

Figure 6. (a) Phase space plots of the SWIs and PUIs at $t=7 \Omega_{c i}^{-1}$ in Run B $($ PUI\% $=10)$. The color shading shows logarithmic distributions of the particle number. (b) The dynamic pressure of SWIs (black) and PUIs (blue), the thermal pressure of SWIs (red) and PUIs (magenta), and the magnetic pressure (green) across the shock front. The black dashed curve indicates the total ion dynamic pressure. The red dashed curve indicates the sum of the total ion thermal pressure and the magnetic pressure. (c) The pressure ratio $\chi=P_{\mathrm{t}}^{\mathrm{PUI}} / P_{\mathrm{t}}$ computed in a downstream region (marked by "DS box" in the top panel).

$\chi$ in the current 2D simulations is about $57.3 \%$ (marked by the horizonal black dashed line).

We perform similar calculations for the high-percentage PUI $(25 \%)$ case, as shown in Figure 7 . In contrast to the lowpercentage PUI (10\%) case, the SWIs lose almost half of their dynamic energy at the PUI foot (from $X=83.75 \mathrm{c} / \omega_{p i}$ to about $\left.X=88 \mathrm{c} / \omega_{p i}\right)$. Consequently, the PUIs gain more thermal energy in this region. The gains of SWI thermal energy and the magnetic energy are lower than those in Run B. Most of the upstream dynamic energy is transferred to PUIs, and the pressure of the PUIs becomes much higher than that of SWIs. In this case, the ratio $\chi$ varies from $61.9 \%$ to $96.3 \%$ along the $Y$ direction due to the remnant effect of the shock front ripples. The average value of the pressure ratio $\chi$ in the downstream region is about $86.6 \%$, which is consistent with previous 1D hybrid models (Wu et al. 2009) and V2 experimental data (Richardson et al. 2008).
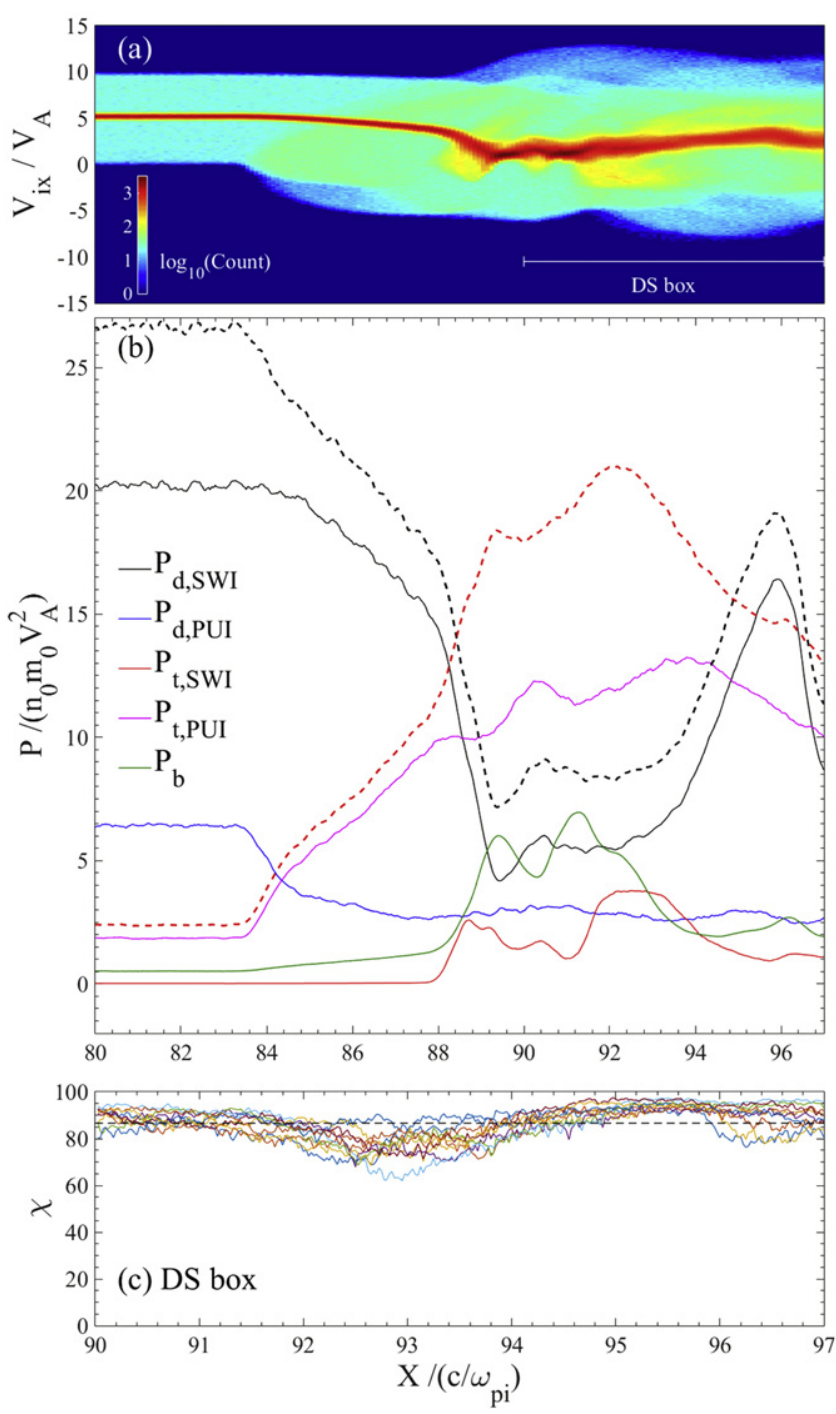

Figure 7. Same format as Figure 6, but for Run C (PUI\% $=25)$.

\section{COMPARISON WITH VOYAGER 2 OBSERVATIONS}

We compare the simulation results with $V 2$ observations. Figure 8(a) shows the observed $B$ field at the TS-3 crossing (after Burlaga et al. 2008). The TS-3 crossing revealed an almost classical perpendicular shock structure. The plasma instrument (Faraday cups) on V2 worked well during the TS crossings. Figure 8(b) shows the observed low-energy ion data. The average speeds corresponding to the channel numbers 1,2 , $3,4,5,6,7,8,9$, and 10 are about $60,90,119,148,216,256$, 300,352 , and $410 \mathrm{~km} \mathrm{~s}^{-1}$, respectively. The bulk speed of the low-energy ions decreases at the foot and reaches a very low value after the shock front. The estimated upstream ion gyroperiod is about 17 minutes (Burlaga et al. 2008). Thus, the time span of the TS-3 crossing plotted in Figure 8 is about $8.8 \Omega_{c i}^{-1}$. The $V 2$ spacecraft moved outwards with a speed of $\sim 18 \mathrm{~km} \mathrm{~s}^{-1}$, and the TS-3 front moved inwards with a speed of $\sim 68 \mathrm{~km} \mathrm{~s}^{-1}$. Hence, the relative speed between $V 2$ and the shock is about $86 \mathrm{~km} \mathrm{~s}^{-1} \sim 2.28 V_{\mathrm{A}}$, where $V_{\mathrm{A}}$ is the Alfvén speed measured upstream of the TS at a distance of 84 AU from the Sun.

In order to generate time series in PIC simulations, a virtual probe ("VP") is used which records the in situ electromagnetic field and plasma information as in previous work for Cluster 

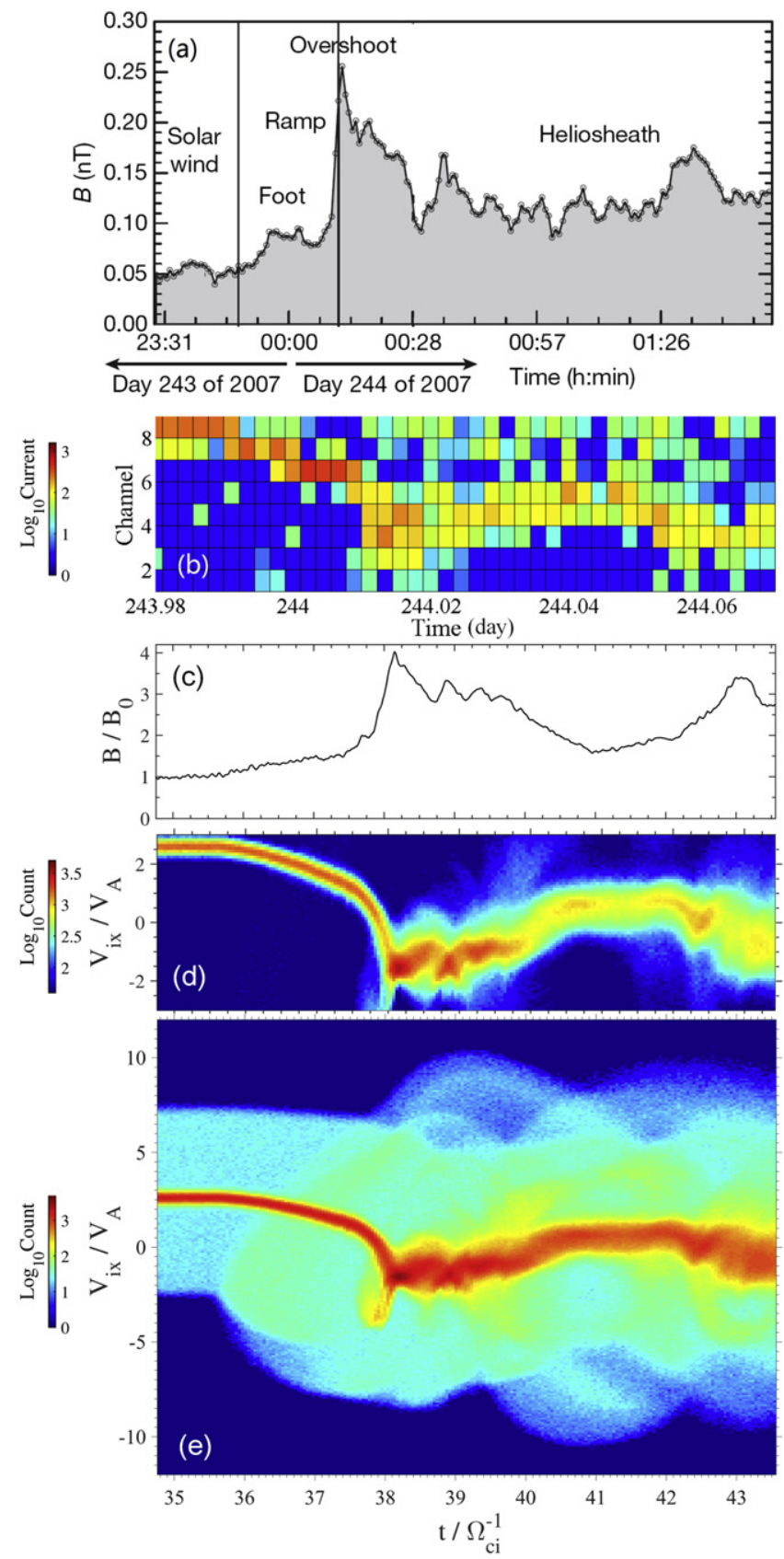

Figure 8. (a) $48 \mathrm{~s}$ averages of the magnetic field strength $B$ at the TS (TS-3, after Burlaga et al. 2008). (b) Velocity distribution observed by Faraday cups on $V 2$. Different channels correspond to different velocity ranges. The color shading shows logarithmic distributions of the current or particle count number. (c) The in situ magnetic field strength $B$ seen by a virtual probe (VP) across the TS profiles obtained from Run C (PUI\% = 25). (d) Corresponding logarithmic distributions of the ions seen by the VP across the TS. Here, the particle counts range from 40 to 5000. (e) Similar plot as in panel (d) but with a full count sensitivity (counts range from 0 to 5000). It shows the complete distribution of the total ions across the TS.

crossings of the Earth's bow shock (Scholer \& Burgess 2006). The VP moves from the upstream to the downstream of the shock in Run C (PUI\% $=25)$. The relative speed between the $\mathrm{VP}$ and the shock front is about $2.2 V_{\mathrm{A}}$. The in situ $B$ field seen by the VP is shown in Figure $8(\mathrm{c})$. The corresponding phase space plot of the ions (SWIs plus PUIs) is shown in Figure $8(d)$. Because the low-energy plasma instrument on $V 2$ is not sensitive enough to see all of the ions (e.g., the wings of the ion velocity distribution), V2 cannot directly observe the PUIs. To imitate this effect, the color bar range of Figure 8(d) has been set from 40 to 5000 , so that ions lower than 40 in the phase space are not seen by the VP. Figure 8(d) shows that the simulated ion distribution across the shock is quite similar to that observed by $V 2$ (Figure $8($ b)). If we set the color bar range from 0 to 5000, then the VP can see all of the ions during the shock crossing (Figure 8(e)). This implies that the thermal speed of the total ions should be higher than that estimated using $V 2$ plasma data. To give a clearer view, the downstream ion velocity distribution function is computed from the overshoot to far downstream of the shock.

Figure 9(a) shows the normalized total ion velocity distribution (black curve) downstream in Run C. The total heliosheath ion distribution function is a superposition of cold, directly transmitted SWIs (DT SWI), hot, reflected SWIs (R SWI), hot, directly transmitted PUIs (DT PUI), and a very hot PUI population (R PUI) that is reflected by the TS and then convected back to the downstream. The highlighted region in red is the part observed by $V 2$. Figure 9(b) shows the composite heliosheath ion distribution function modeled by Zank et al. (2010). The form of their total ion distribution function is similar to that obtained from our PIC simulations (Figure 9(a)). For reference, the blue solid and red dashed curves illustrate a $\kappa$ distribution and a Maxwellian distribution with the same downstream density and temperature. Both the modeled composite distribution and the $\kappa$ distribution have more ions in the wings of the distribution than a Maxwellian. The R SWI population is not included in Zank's model; our simulations show that the contribution of R SWIs is small compared with that of R PUIs. Previous 1D PIC simulations have also shown that the fraction of R SWIs decreases with PUI\% at the shock (Yang et al. 2012a). Figure 9(c) shows the ion velocity distribution observed by the Faraday cup on V2 in the heliosheath near the TS. Compared with the experimental data, the velocity distribution functions obtained from both the PIC simulations and Zank's models imply that $V 2$ only saw the tip of the iceberg (i.e., the cool core of the total distribution). From this cool part of the distribution, one can determine the bulk velocity of the plasma, the number density of SWIs, and roughly the temperature of SWIs.

\section{CONCLUSIONS AND DISCUSSION}

In this paper, we use a 2D PIC code to investigate the impact of PUIs on the nonstationarity and energy dissipation of the TS. We summarize our main findings below.

1. In contrast to $1 \mathrm{D}$ simulations, we show that shock front ripples form even when the relative percentage of PUIs is $25 \%$. The excitation of ripples is associated with the reflected SWIs. In a high-percentage $(25 \%)$ PUI case, the gyroradius of the reflected SWIs becomes smaller due to the enhanced local $B$ caused by the reflected PUIs at the shock foot. This leads to a narrower ripple excitation region at the shock front. The period of the shock front nonstationarity caused by the ripples is about $1-2 \Omega_{c i}^{-1}$. The multiple crossings of the TS in a short time duration observed at TS-2 can be explained by the shock front nonstationarity.

2. The energy dissipation process is examined for shocks with different percentages of PUIs. At a shock with no 

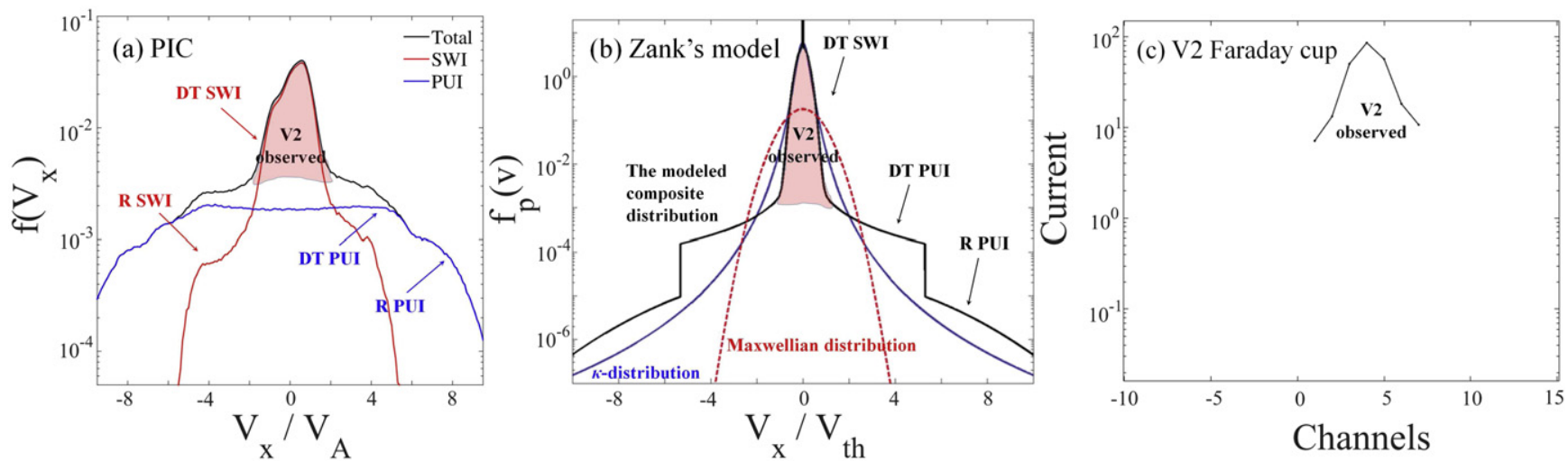

Figure 9. (a) Velocity distribution of the ions (black) selected in the "DS box" (see Figure 7) of the TS. Contributions of SWIs and PUIs to the total distribution are indicated by red and blue curves, respectively. The highlighted region (cool core) at the peak of the distribution is expected to be observed by V2. (b) Heliosheath particle distribution (black) modeled by Zank et al. (2010). The Maxwellian (red) and $\kappa$ (blue) distributions with the same temperature and particle density are also shown for reference. (c) Cool core of the ion distribution observed by V2 in the downstream region of TS-3.

PUIs, the dynamic energy is transferred to the thermal energy of SWIs and to the magnetic energy. For a shock with $25 \%$ PUIs, most of the dynamic energy is transferred to the thermal energy of PUIs instead of the SWIs.

3. In order to examine the effect of PUIs on the energy partition downstream of the shock, we compute the pressure ratio of PUIs to SWIs $\chi$. In the $10 \%$ PUI case, the ratio is $57.3 \%$. For the $25 \%$ PUI case, the average value of $\chi$ is $86.6 \%$ and varies from $61.9 \%$ to $96.3 \%$ along the $Y$ direction due to the remnant effect of shock front ripples. In 1D simulations, the ratio $\chi$ cannot vary along the $Y$ direction because $\partial / \partial Y=0$. The $y$-averaged ratio $\bar{\chi}$ obtained in the $2 \mathrm{D}$ simulation is consistent with that obtained in previous 1D hybrid simulations and with that estimated from the Voyager 2 experimental data.

4. We compare our 2D PIC simulations with the magnetic field and plasma observations for a typical shock crossing (TS-3). The velocity distribution of low energy SWIs resulting from PIC simulations are quite similar to the Voyager 2 observed plasma data. In addition, the velocity distribution of the PUIs, which cannot be directly observed by Voyager 2, is also predicted for use in future observations and studies.

5. A composite heliosheath ion distribution function is obtained in our simulation. The core of the distribution function is formed by the cool directly transmitted SWIs, the shoulder is contributed by both the hot directly transmitted PUIs and the reflected SWIs, and the wing of the distribution is dominated by the very hot reflected PUIs. The shape of the total distribution is similar to the theoretical model made by Zank et al. (2010). Compared with the experimental data, both of the velocity distribution functions obtained from the PIC simulations and Zank's model imply that Voyager 2 only observed the tip of the iceberg (i.e., the cool core of the total distribution). The PIC simulation results may help interpret the IBEX data in addition to probing the microphysics of the TS.

By using a fixed shock profile, Burrows et al. (2010) found that multiply reflected PUIs (i.e., shock surfing accelerated PUIs) could account for the TS downstream energy gains that are generally assumed to go into the PUIs. We find that the TS is nonstationary and the shock front width changes with time.
Shock surfing may be sufficient to heat the PUIs only when the TS is steep and has a narrow shock profile (Lee et al. 1996; Zank et al. 1996; Lipatov \& Zank 1999; Shapiro \& Üçer 2003; Yang et al. 2009).

The authors are grateful to B. Lembège and F. Guo for helpful discussions. This research was supported by NSFC under grants No. 41204106 and 41374173, the Recruitment Program of Global Experts of China, the Specialized Research Fund for State Key Laboratories of China (Y32612A24S, Y5262AA52S), and the Shanghai Science Foundation (12ZR1451500).

\section{REFERENCES}

Axford, W. I., Lee, I. E., \& Skadron, G. 1977, in Proc. 15th ICRC (Plovdiv), 11,132

Bell, A. R. 1978, MNRAS, 182, 147

Birdsall, C. K., \& Langdon, A. B. 1991, Plasma Physics via Computer Simulations (New York: McGraw-Hill)

Blandford, R. D., \& Ostriker, J. P. 1978, ApJL, 221, L29

Buneman, O., Nishikawa, K.-I., \& Neubert, T. 1995, in Space Plasmas: Coupling Between Small and Medium Scale Processes, ed. M. AshourAbdalla, T. Chang, \& P. Dusenbery (AGU Geophys. Monograph 86; Washington, DC: AGU), 347

Burgess, D., Lucek, E. A., Scholer, M., et al. 2005, SSRv, 118, 205

Burgess, D., \& Scholer, M. 2007, PhPl, 14, 012108

Burlaga, L. F., Ness, N. F., Acuña, M. H., et al. 2008, Natur, 454, 75

Burrows, R. H., Zank, G. P., Webb, G. M., et al. 2010, ApJ, 715, 1109

Cai, D. S., Li, Y. T., Nishikawa, K., et al. 2003, Space Plasma Simulation (New York: Springer)

Caprioli, D., \& Spitkovsky, A. 2014, ApJ, 783, 91

Chapman, S. C., Lee, R. E., \& Dendy, R. O. 2005, SSRv, 121, 5

Decker, R. B., Krimigis, S. M., Roelof, E. C., et al. 2005, Sci, 309, 2020

Decker, R. B., Krimigis, S. M., Roelof, E. C., et al. 2008, Natur, 453, 67

Fisk, L. A., Gloeckler, G., \& Zurbuchen, T. H. 2006, ApJ, 644, 631

Guo, F., \& Giacalone, J. 2010, ApJ, 715, 406

Guo, F., \& Giacalone, J. 2012, ApJ, 753, 28

Guo, F., \& Giacalone, J. 2013, ApJ, 773, 158

Galeev, A. A., \& Sagdeev, R. Z. 1988, Ap\&SS, 144, 427

Giacalone, J. 2005, ApJL, 628, L37

Gloeckler, G., \& Geiss, J. 1998, SSRv, 86, 127

Goncharov, O., Šafránková, J., Němeček, Z., et al. 2014, GeoRL, 41, 8100

Hada, T., Oonishi, M., Lembège, B., et al. 2003, JGR, 108, 1233

Hao, Y. F., Lu, Q. M., Gao, X. L., et al. 2014, JGR, 119, 3225

Heerikhuisen, J., Pogorelov, N. V., Florinski, V., et al. 2008, ApJ, 682,679

Hellinger, P., Mangeney, A., \& Matthews, A. 1996, GeoRL, 23, 621

Hellinger, P., \& Trávnícek, P. 2002, GeoRL, 29, 87

Jokipii, J. R. 2008, Natur, 454, 38 
Lee, M. A., \& Ip, W. H. 1987, JGR, 92, 11041

Lee, M. A., Shapiro, V. D., \& Sagdeev, R. Z. 1996, JGR, 101, 4777

Lee, R. E., Chapman, S. C., \& Dendy, R. O. 2005a, PhPl, 12, 012901

Lee, R. E., Chapman, S. C., \& Dendy, R. O. 2005b, AnGeo, 23, 643

Lembège, B., \& Dawson, J. M. 1987, PhFl, 30, 1767

Lembège, B., Giacalone, J., Scholer, M., et al. 2004, SSRv, 110, 161

Lembège, B., Savoini, P., Hellinger, P., et al. 2009, JGR, 114, A03217

Liewer, P. C., \& Goldsten, B. E. 1993, JGR, 98, 15211

Lipatov, A. S., \& Zank, G. P. 1999, PhRvL, 82, 18

Liu, K. J., Gary, S. P., \& Winske, D. 2010, JGR, 115, A12114

Liu, Y., Richardson, J. D., Belcher, J. W., et al. 2006, JGR, 111, A09108

Liu, Y., Richardson, J. D., Belcher, J. W., et al. 2007, ApJL, 659, L65

Lu, Q. M., \& Wang, S. 2006, JGR, 111, A05204

Matsukiyo, S., \& Scholer, M. 2011, JGR, 116, A08106

Matsukiyo, S., \& Scholer, M. 2014, JGR, 119, 2388

Matsukiyo, S., Scholer, M., \& Burgess, D. 2007, AnGeo, 25, 283

Mazelle, C., Lembège, B., Morgenthaler, A., et al. 2010, in AIP Conf. Proc. 1216, 12th Intl. Solar Wind Conf., ed. M. Maksimovic et al. (Melville, NY: AIP), 471

McComas, D. J., Allegrini, F., Bochsler, P., et al. 2009, Sci, 326, 959

Möbius, E., Hovestadt, D., Klecker, B., et al. 1985, Natur, 318, 426

Nishikawa, K. 1997, JGR, 102, 17631

Nishimura, K., Matsukiyo, H., \& Kojima, H. 2003, JGR, 108, 1182

Oka, M., Zank, G. P., Burrows, R. H., et al. 2011, in AIP Conf. Proc. 1366,

Partially Ionized Plasmas Throughout the Cosmos-Proc. 2010 Huntsville workshop, ed. V. Florinski et al. (Melville, NY: AIP), 53

Rekaa, V. L., Chapman, S. C., \& Dendy, R. O. 2014, ApJ, 791, 26

Richardson, J. D., Kasper, J. C., Wang, C., et al. 2008, Natur, 454, 63
Richardson, J. D., \& Liu, Y. 2007, in AIP Conf. Proc. 932, Turbulence and Nonlinear Processes in Astrophysical Plasmas, 6th Annual Intl. Astrophysics Conf., ed. D. Shaikh \& G. P. Zank (Melville, NY: AIP), 387 Savoini, P., \& Lembège, B. 1994, JGR, 99, 6609

Scholer, M., \& Burgess, D. 2006, PhPl, 13, 062101

Scholer, M., Shinohara, I., \& Matsukiyo, S. 2003, JGR, 108, 1014

Shapiro, V. D., \& Üçer, D. 2003, P\&SS, 51, 665

Spitkovsky, A. 2005, in AIP Conf. Proc. 801, Astrophysical Source of High

Energy Particles and Radiation, ed. T. Bulik, B. Rudak, \& G. Madejski (Melville, NY: AIP), 345

Spitkovsky, A. 2008, ApJL, 682, L5

Stone, E. C., Cummings, A. C., McDonald, F. B., et al. 2008, Natur, 454, 71

Su, Y. Q., Lu, Q. M., Gao, X. L., et al. 2012, PhPl, 19, 092108

Thomas, V. A. 1989, JGR, 94, 12009

Tiu, D., Cairns, I. H., Yuan, X. Q., et al. 2011, JGR, 116, A04228

Vasyliunas, V. M., \& Siscoe, G. L. 1976, JGR, 81, 1247

Villasenor, J., \& Buneman, O. 1992, CoPhC, 69, 306

Whang, Y. C., \& Burlaga, L. F. 2000, GeoRL, 27, 1607

Winske, D., \& Quest, K. B. 1988, JGR, 91, 9681

Wu, P., Winske, D., Gary, S. P., et al. 2009, JGR, 114, A08103

Yang, Z. W., Han, D. S., Yang, H. G., et al. 2012a, Ap\&SS, 341, 241

Yang, Z. W., Lu, Q. M., Gao, X. L., et al. 2013, PhPl, 20, 092116

Yang, Z. W., Lu, Q. M., \& Lembège, B. 2012b, JGR, 117, A07222

Yang, Z. W., Lu, Q. M., Lembège, B., et al. 2009, JGR, 114, A03111

Yuan, X. Q., Cairns, I. H., Trichtchenko, L., et al. 2009, GeoRL, 36, L05103

Zank, G. P., Heerikhuisen, J., Pogorelov, N. V., et al. 2010, ApJ, 708, 1092

Zank, G. P., Li, G., Florinski, V., et al. 2006, JGR, 111, A06108

Zank, G. P., Pauls, H. L., Carins, I. H., \& Webb, G. M. 1996, JGR, 101, 457 